Supporting Information

\title{
Zn-doped Poly Heptazine Imides Integrated with a van der Waals Homojunction Boosting Visible Light Driven Water Oxidation Activities
}

Zhiming Pan ${ }^{1,2}$, Meng Zhao ${ }^{1}$, Hangyu Zhuzhang, ${ }^{1}$ Guigang Zhang ${ }^{1 *}$, Masakazu Anpo ${ }^{1}$, and Xinchen Wang ${ }^{1 *}$

${ }^{1}$ State Key Laboratory of Photocatalysis on Energy and Environment, College of Chemistry, Fuzhou University, Fuzhou 350116, P. R. China

${ }^{2}$ College of Chemical Engineering, Fuzhou University, Fuzhou 350116, P. R. China

* Corresponding author: Prof. Guigang Zhang and Prof. Xinchen Wang

Email: guigang.zhang@fzu.edu.cn (G. Zhang) and xcwang@fzu.edu.cn (X. Wang)

Homepage: http://wanglab.fzu.edu.cn 


\section{Supporting Information Content}

1. Experimental procedures....................................................

2. types of VDW junction.................................................. 6

3. Morphology characterization............................................6

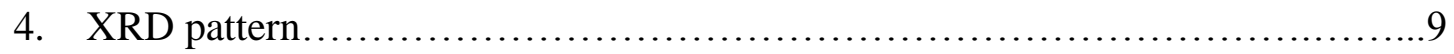

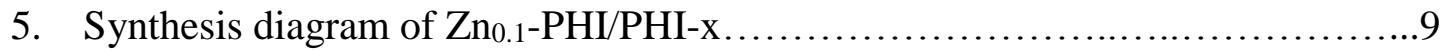

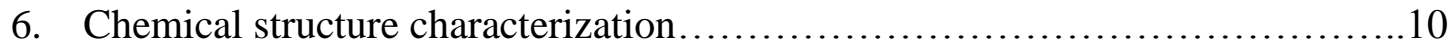

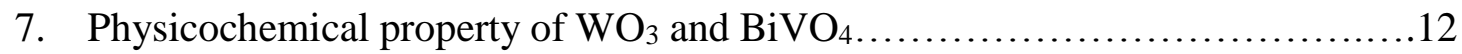

8. Photocatalytic water oxidation experiment.................................. 13

9. Physicochemical characterizations of after reaction of $\mathrm{Zn}_{0.2}-\mathrm{PHI} / \mathrm{PHI} \ldots \ldots \ldots \ldots \ldots 15$

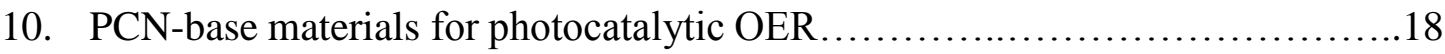

11. Optical characterization............................................. 19

12. Mott-Schottky curves...............................................20

13. EIS fitted parameters of PHI and $\mathrm{Zn}_{0.2}$-PHI/PHI catalyst..................20

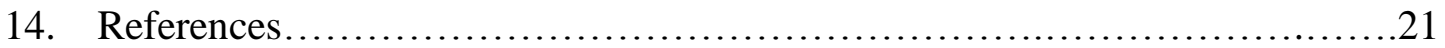




\section{Experimental procedures}

Material: $\mathrm{LiCl}(99 \%), \mathrm{KCl}(99 \%), \mathrm{ZnCl}_{2}(98 \%), \mathrm{FeCl}_{3}(98 \%)$, and melamine $\left(\mathrm{C}_{3} \mathrm{H}_{6} \mathrm{~N}_{6}\right.$, 99\%) were obtained from Sinopharm Chemical Reagent Co.

Synthesis of PCN catalysts: $5 \mathrm{~g}$ of $\mathrm{C}_{6} \mathrm{H}_{6} \mathrm{~N}_{6}$ was placed into a porcelain crucible and thermal heated at $823 \mathrm{~K}$ for $4 \mathrm{~h}$ in the air atmosphere with a ramping rate of $2.3 \mathrm{~K} \mathrm{~min}^{-}$

1. The product is labeled as PCN for simplicity.

Synthesis of PHI catalysts: $1 \mathrm{~g}$ PCN, $5.5 \mathrm{~g} \mathrm{KCl}$ and $4.5 \mathrm{~g} \mathrm{LiCl}$ salts were thoroughly ground in a mortar to generate a homogeneous mixture. Then, the resultant solids were transferred into a porcelain crucible and directly heated to $823 \mathrm{~K}$ for $4 \mathrm{~h}$ in the $\mathrm{N}_{2}$ atmosphere with a ramping rate of $2.3 \mathrm{~K} \mathrm{~min}^{-1}$. When the sample was naturally cooled down to room temperature, the obtained solids were collected and thoroughly washed with ultrapure water to remove excess amounts of metal salts and dried at $333 \mathrm{~K}$ for 12 $\mathrm{h}$ under vacuum. The product is labeled as PHI for simplicity.

Synthesis of $\mathbf{Z n} \mathbf{n}_{\mathbf{x}}-\mathbf{P H I} / \mathbf{P H I}$ catalysts: The procedure is very similar to that of the synthesis procedure of PHI. The only difference is a certain amount of $\mathrm{ZnCl}_{2}$ was added into the mixture of $\mathrm{LiCl}$ and $\mathrm{KCl}$ to form ternary melting salts. The final products were named as $\mathrm{Zn}_{0.05}$-PHI/PHI ( $\left.\mathrm{x}=0.05\right), \mathrm{Zn}_{0.1}$-PHI/PHI ( $\left.\mathrm{x}=0.1\right), \mathrm{Zn}_{0.2}-\mathrm{PHI} / \mathrm{PHI}(\mathrm{x}=0.2)$ and $\mathrm{Zn}_{0.4}$-PHI/PHI ( $\left.=0.4\right)$, where $\mathrm{x}(\mathrm{x}=0.05 \mathrm{~g}, 0.1 \mathrm{~g}, 0.2 \mathrm{~g}$ and $0.4 \mathrm{~g})$ is initial weight amounts of $\mathrm{ZnCl}_{2}$ salts. 
Characterization: Transmission electron microscopy (TEM) and high-resolution transmission electron microscopy (HRTEM) images were gained on a FEI TECNAI G2F20 instrument. Field-emission scanning electron microscope (SEM) were performed on a Hitachi New Generation SU8010 field emission scanning electron microscope. The $\mathrm{N}_{2}$ adsorption-desorption isotherms was obtained on a Micromeritics ASAP 2020 system at $77 \mathrm{~K}$. The Brunauer-Emmett-Teller (BET) was used to test the specific surface area of the PCN, PHI and $\mathrm{Zn}_{0.2}-\mathrm{PHI} / \mathrm{PHI}$ catalysts. Inductively coupled plasma atomic emission spectrometry measurements were performed using an iCAP7400 instrument to detect concentrations of Zn. The powder X-ray diffraction (XRD) patterns were carried out on a Bruker D8 Advance diffractometer. Attrnuated total internal reflectance Fourier transform infrared spectroscopy (ATR-FTIR) spectra were gained by using a Nicolet Magna 670 FTIR spectrometer, scanning from 4000 to $400 \mathrm{~cm}^{-1}$. X-Ray photoelectron spectroscopy (XPS) spectra were obtained by using a Thermo Scientific ESCALAB250 instrument. Solid-state ${ }^{13} \mathrm{C}$ NMR spectra were obtained on a Bruker Advance 500 spectrometer. Photoluminescence (PL) spectra were performed in Edinburgh FL/FSTCSPC 920 spectrophotometer. The integrated PL peak intensity increases monotonically, and the corresponding $\mathrm{E}_{\mathrm{b}}$ could be experimentally gained by fitting these data using the Arrhenius equation, $\mathrm{I}(\mathrm{T})=\mathrm{I}_{0} /\left(1+\mathrm{A} \exp \left(-\mathrm{E}_{\mathrm{b}} / \mathrm{k}_{\mathrm{B}} \mathrm{T}\right)\right){ }^{1}$ $\mathrm{I}_{0}$ is the intensity at $0 \mathrm{~K}, \mathrm{k}_{\mathrm{B}}$ is the Boltzmann constant, $\mathrm{E}_{\mathrm{b}}$ is exciton binding energy. UV-Vis diffuse reflectance spectra (DRS) were obtained from a Varian Cary 500 Scan UV-visible system. Electron paramagnetic resonance (EPR) spectra were obtained from a Bruker model A 300 spectrometer. Electrochemical measurements were conducted -S4- 
on a BioLogic VSP-300 electrochemical System. Three-electrode cell was used, including an electrolyte solution $0.2 \mathrm{M} \mathrm{NaSO}_{4}(\mathrm{pH}=6.8)$, a $\mathrm{Pt}$ foil as the counter electrode, a saturated calomel electrode (SCE) as reference electrode, and a working electrode. The working electrode was prepared by coating of PHI or $\mathrm{Zn}_{0.2}-\mathrm{PHI} / \mathrm{PHI}$ catalyst films on $0.5^{*} 0.5 \mathrm{~cm}^{-2}$ pieces FTO glass $\left(0.4 \mathrm{mg} \mathrm{cm}^{-2}\right)$. The work electrode was then air-dried at $298 \mathrm{~K}$. The epoxy was then used to cover all surface except for the catalysts films. Electrochemical impedance spectroscopy (EIS) was measured at $-0.4 \mathrm{~V}$ vs SCE from $1 \mathrm{kHz}$ to $200 \mathrm{mHz}$ by applying an amplitude of $10 \mathrm{mV}$ in $0.2 \mathrm{M} \mathrm{NaSO}_{4}$ solution.

Photocatalytic water splitting tests: For photocatalytic water oxidation reaction, 50 mg of PCN, PHI, $\mathrm{Zn}_{x}-\mathrm{PHI} / \mathrm{PHI}, \mathrm{WO}_{3}$ or $\mathrm{BiVO}_{4}$ catalysts were dispersed in an aqueous solution $(100 \mathrm{~mL})$ containing $\mathrm{FeCl}_{3}(81 \mathrm{mg})$ as electron sacrificial agent (or containing $0.17 \mathrm{~g} \mathrm{AgNO}_{3}$ as electron sacrificial agent and $0.2 \mathrm{~g} \mathrm{La}_{2} \mathrm{O}_{3}$ as $\mathrm{pH}$ buffer agent). Then, the photocatalytic vessel was evacuated five times to eliminate air before irradiation under a $300 \mathrm{~W}$ Xe lamp with a working current of 15A and a water-cooling filter (285 $\mathrm{K})$. The wavelength of the incident light was controlled by applying some proper long pass cut-off filter. In addition, the obtained gases were tested by gas chromatography equipped with a thermal conductive detector, using Argon as the carrier gas. 
Type-I: straddle alignment

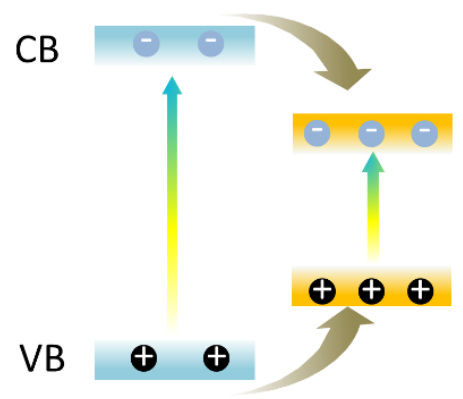

Type-II: staggered alignment

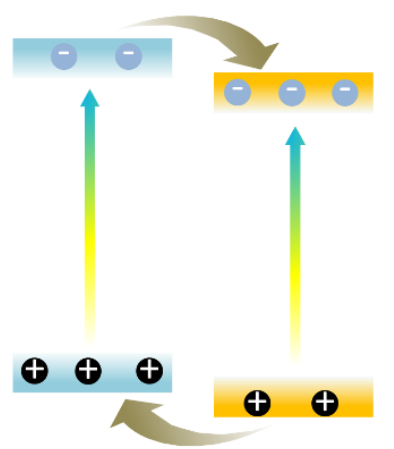

Type-III: broken gap

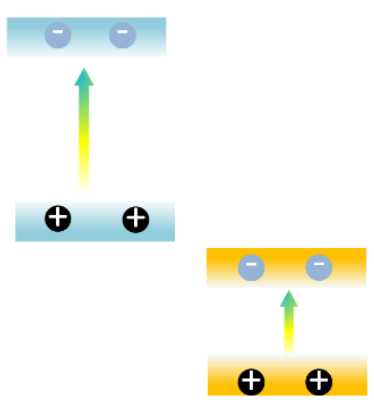

Scheme S1. Three types of VDW junction organized by band alignment. CB: conduction band; VB: valence band.

\section{Morphology characterization}
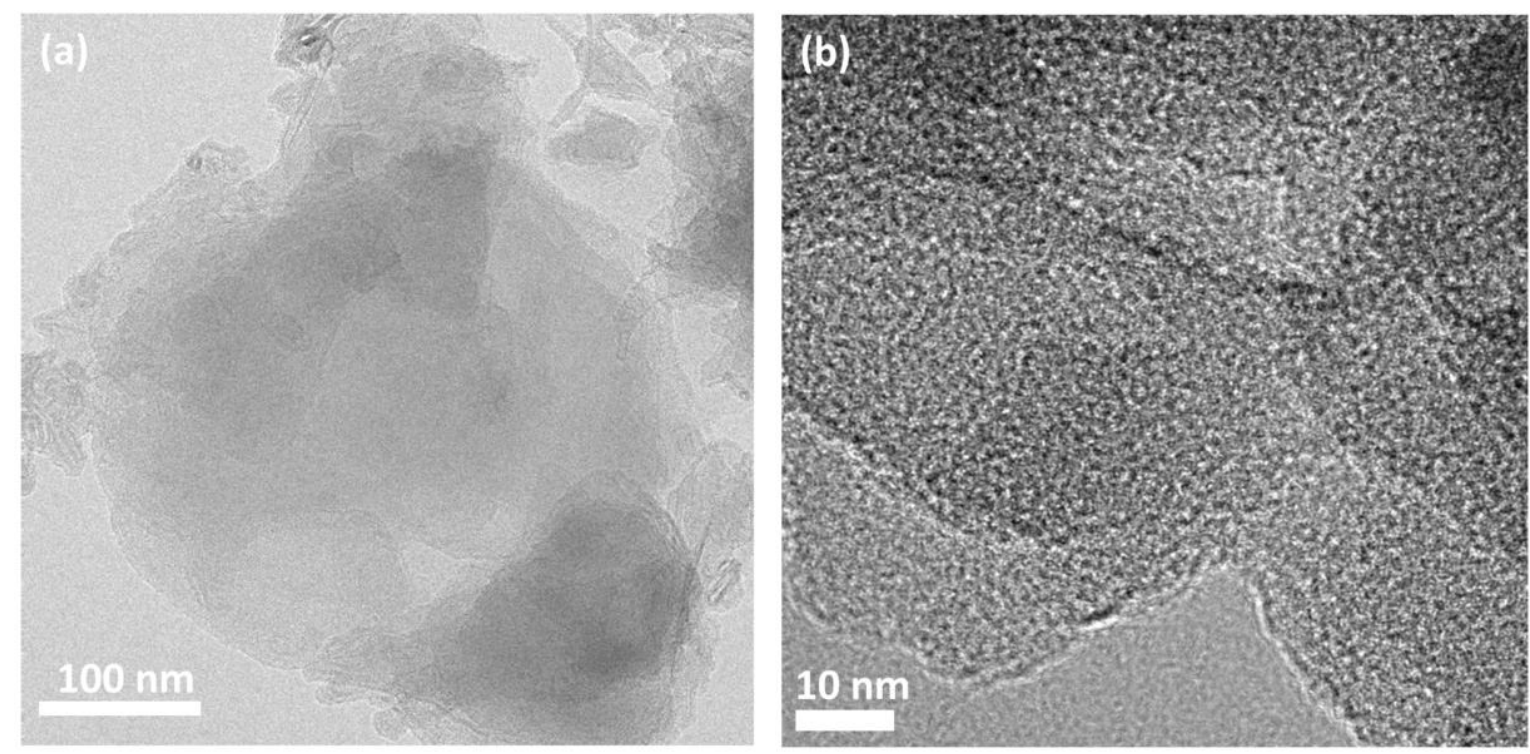

Figure S1. (a)TEM and (b) HRTEM images of PCN catalyst. 

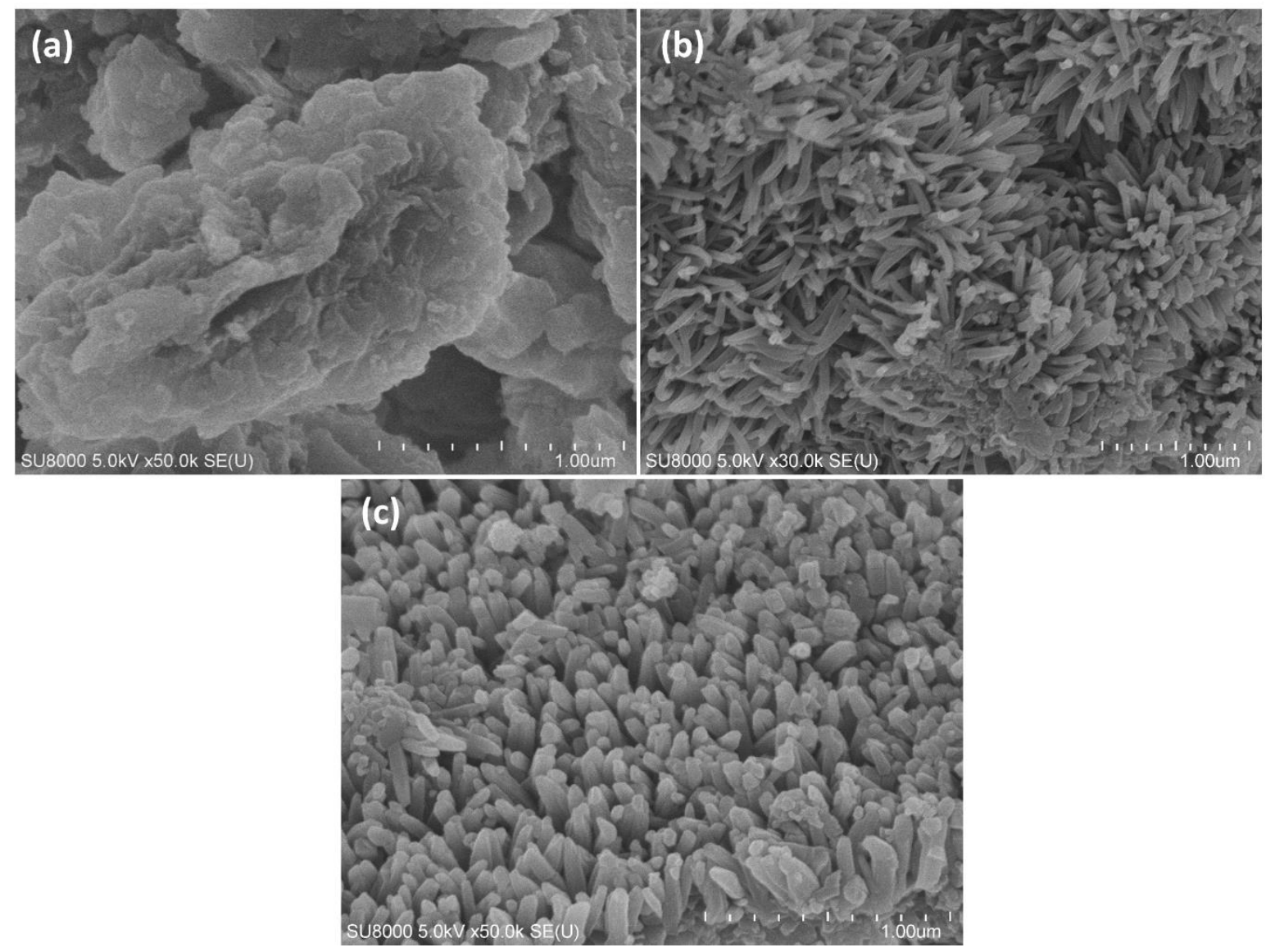

Figure S2. SEM images of (a) PCN, (b) PHI and (c) $\mathrm{Zn}_{0.2}-\mathrm{PHI} / \mathrm{PHI}$ catalysts. 


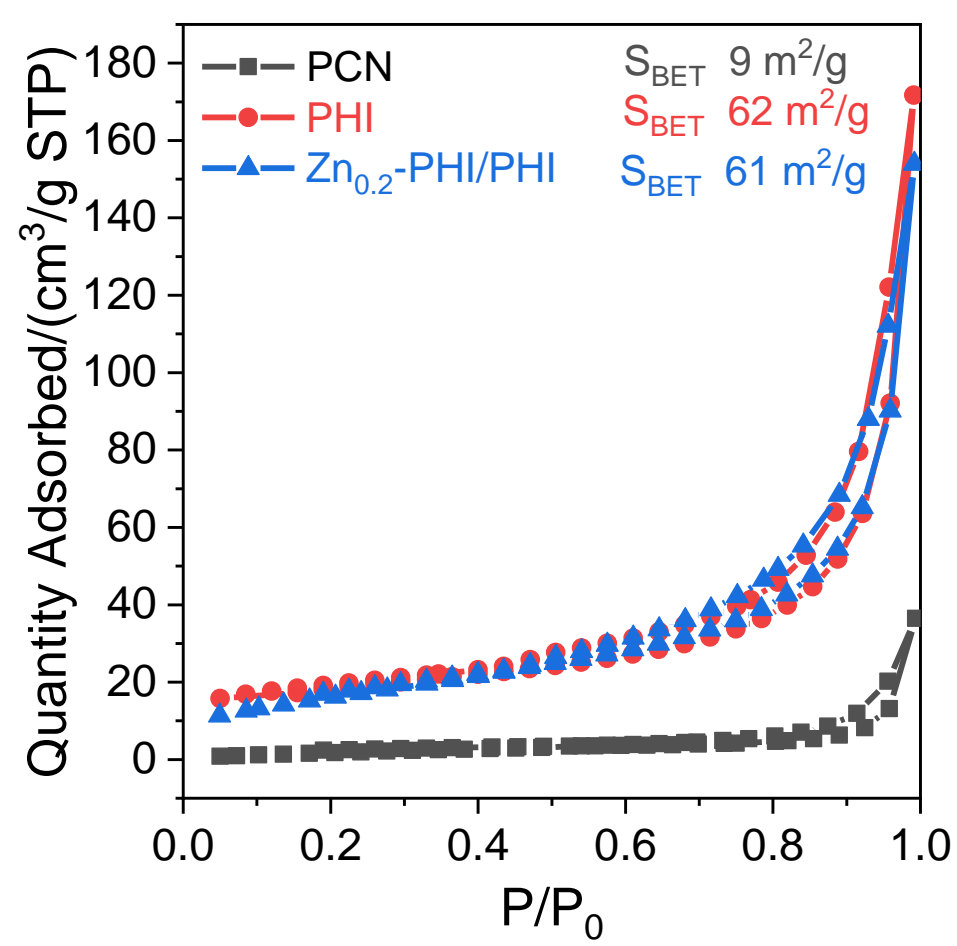

Figure S3. Nitrogen adsorption-desorption isotherms of $\mathrm{PCN}, \mathrm{PHI}$ and $\mathrm{Zn}_{0.2}-\mathrm{PHI} / \mathrm{PHI}$ catalysts.

Table S1. Physical properties of PCN, PHI and $\mathrm{Zn}_{0.2}-\mathrm{PHI} / \mathrm{PHI}$ catalysts.

\begin{tabular}{c|c|c}
\hline sample & $\begin{array}{c}\text { Surface area } \\
{\left[\mathrm{m}^{2} \cdot \mathrm{g}^{-1}\right]}\end{array}$ & $\begin{array}{c}\text { Pore volume } \\
{\left[\mathrm{cm}^{3} \cdot \mathrm{g}^{-1}\right]}\end{array}$ \\
\hline PCN & 9 & 0.027 \\
\hline PHI & 62 & 0.26 \\
\hline $\mathrm{Zn}_{0.2}$ PHI/PHI & 61 & 0.24 \\
\hline
\end{tabular}




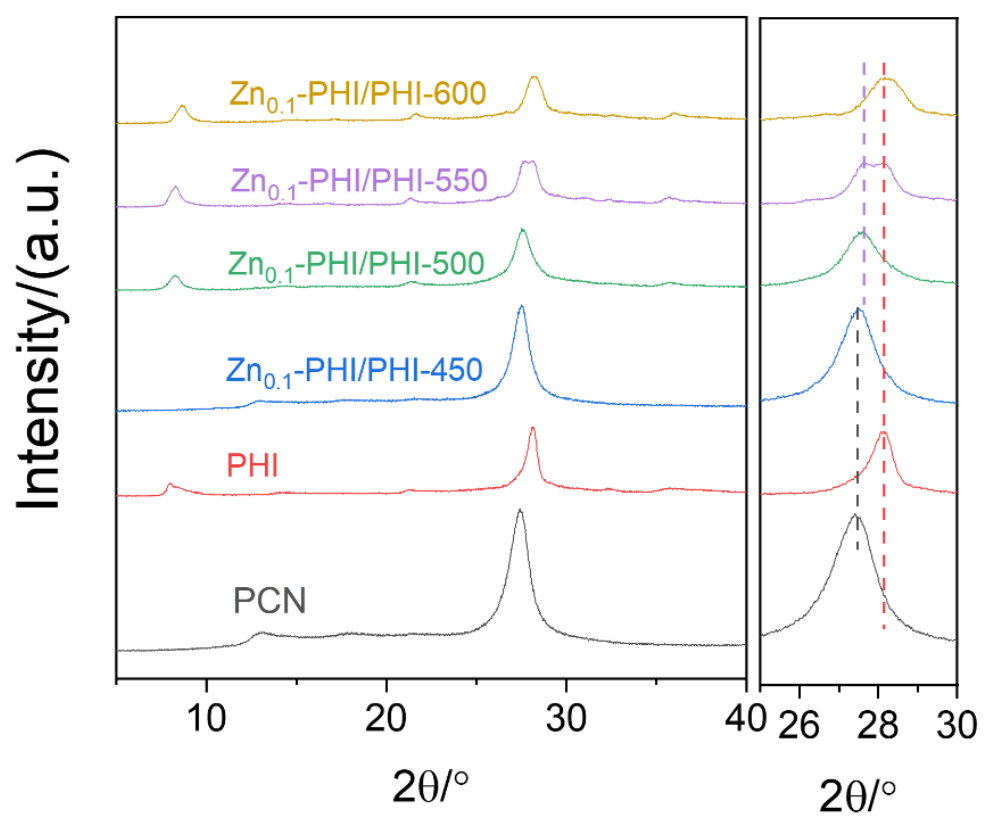

Figure S4. Powder-XRD pattern of $\mathrm{Zn}_{0.1}-\mathrm{PHI} / \mathrm{PHI}-\mathrm{x}\left(\mathrm{x}=450,500,550\right.$ and $\left.600{ }^{\circ} \mathrm{C}\right)$.

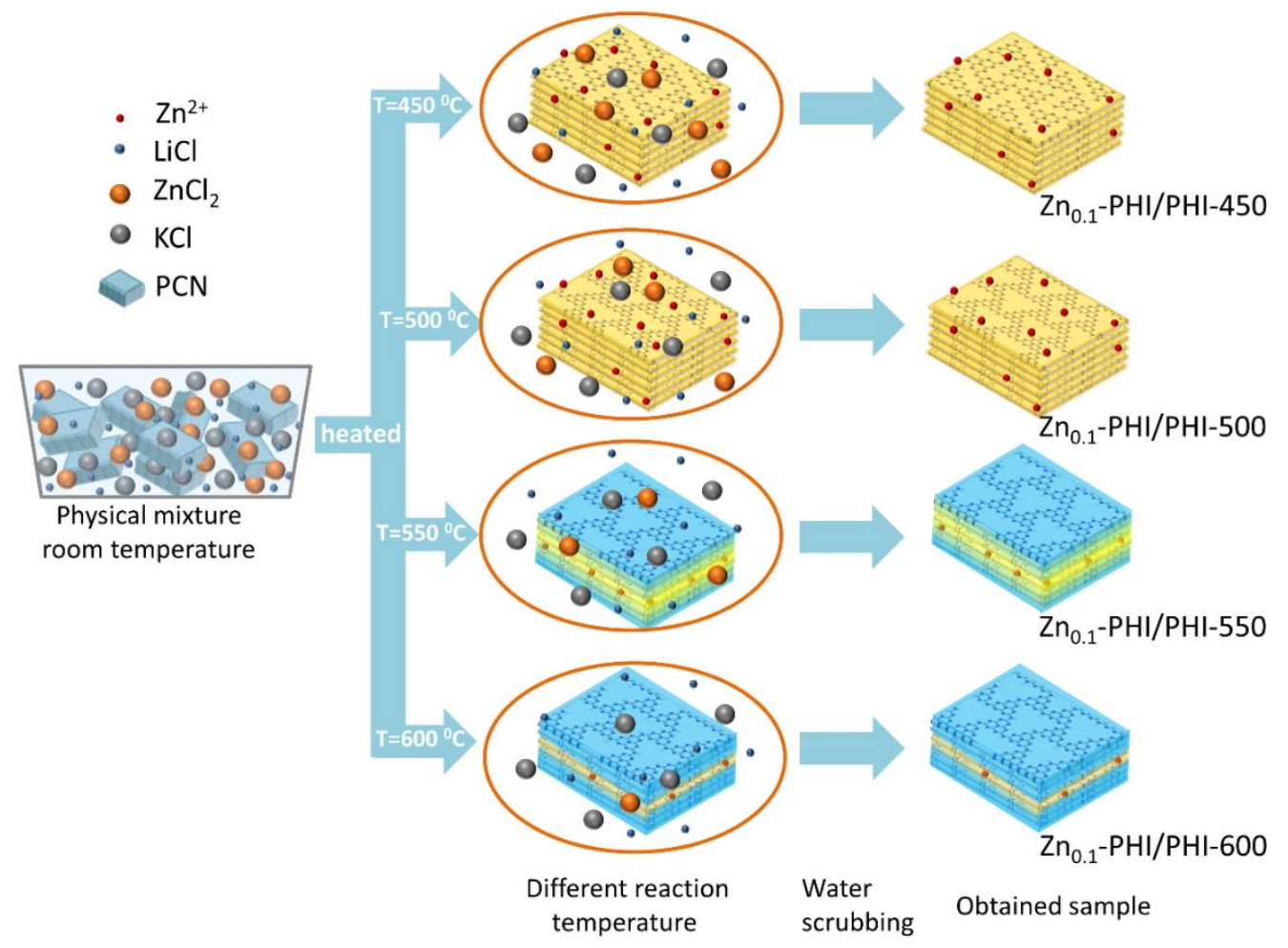

Figure S5. Proposed synthetic process of $\mathrm{Zn}_{0.1}-\mathrm{PHI} / \mathrm{PHI}-\mathrm{x}(\mathrm{x}=450,500,550$ and $\left.600^{\circ} \mathrm{C}\right)$ 


\section{Chemical structure characterization}

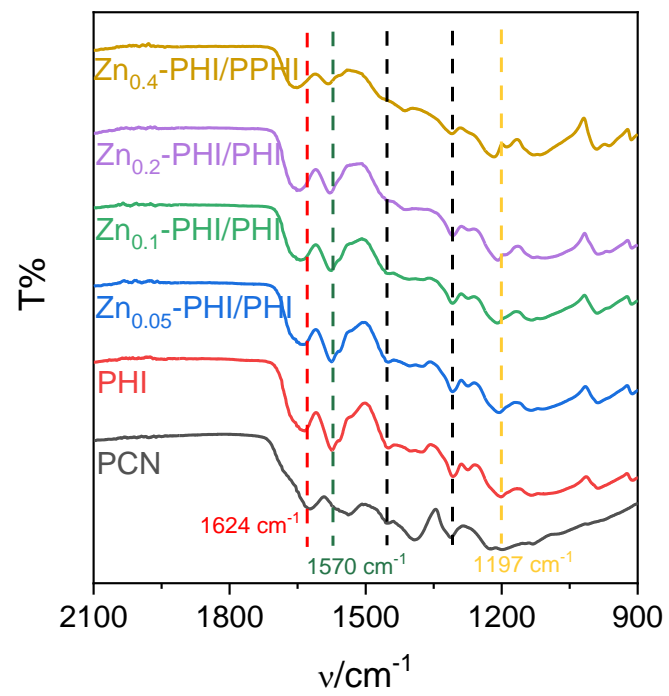

Figure S6. ATR-FTIR spectra of PCN, PHI and $\mathrm{Zn}_{\mathrm{x}}-\mathrm{PHI} / \mathrm{PHI}$ catalysts. 

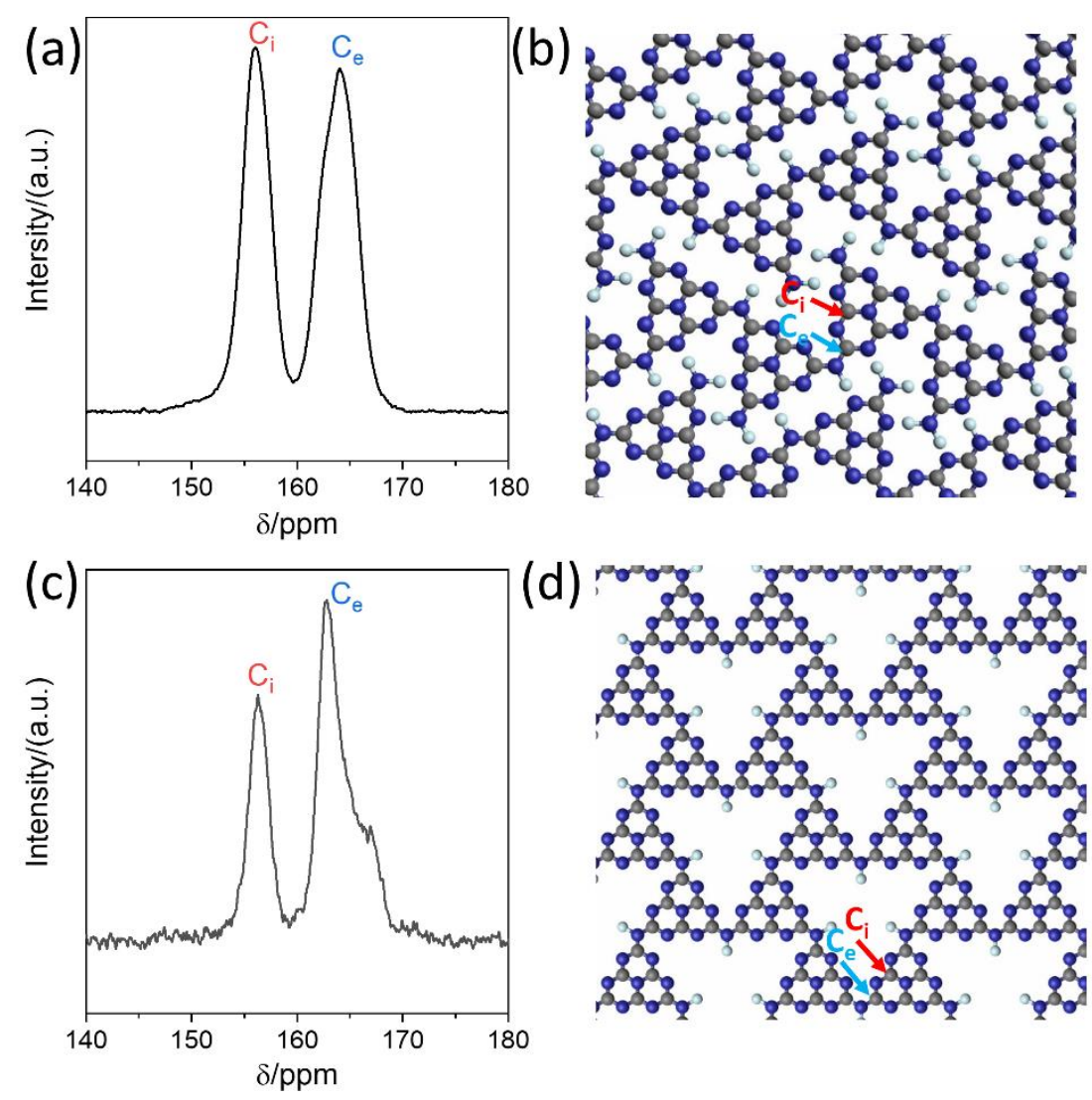

(d)
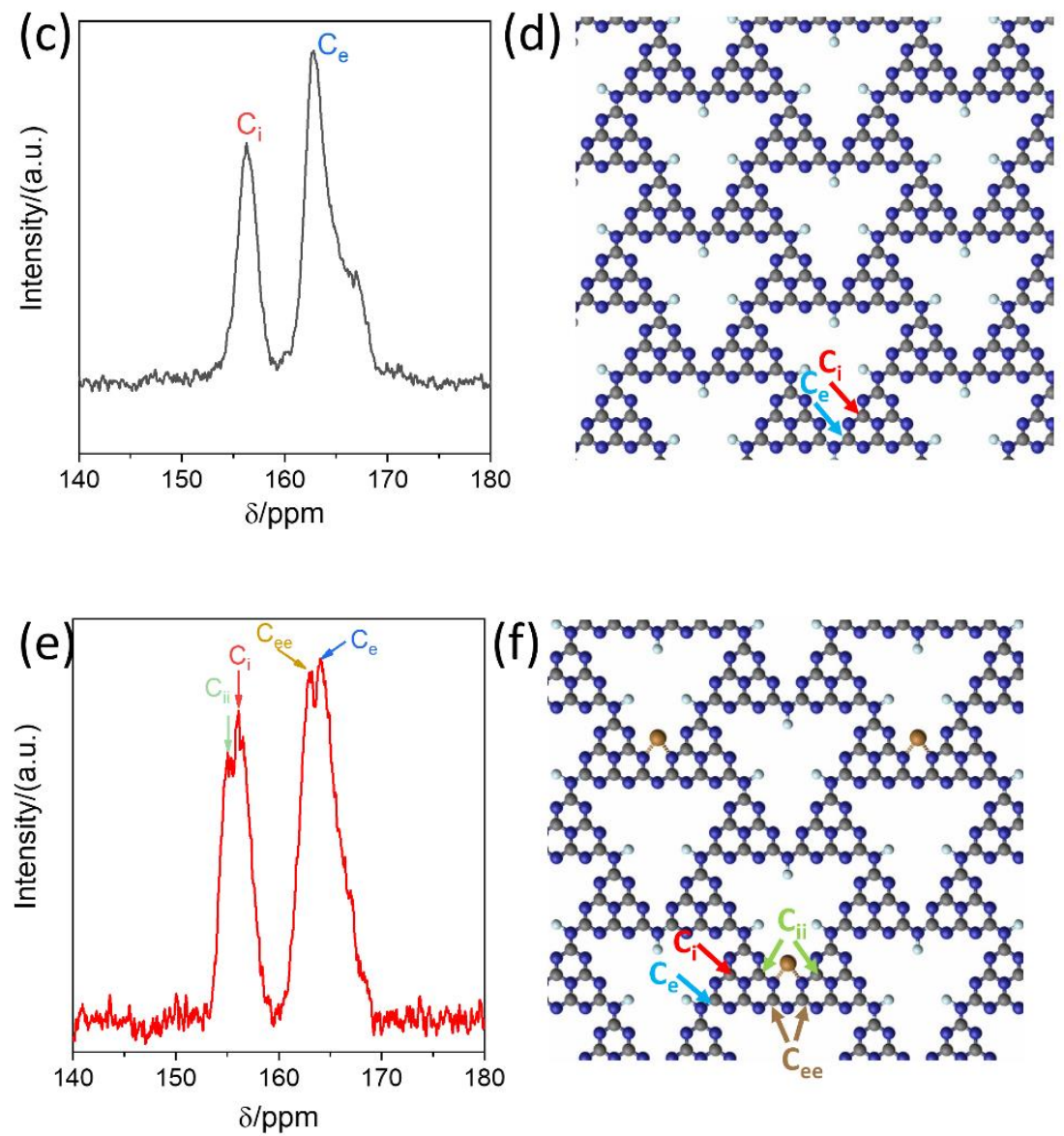

Figure S7. Solid state ${ }^{13} \mathrm{C}$ NMR spectra of (a) PCN, (c) PHI and (e) $\mathrm{Zn}_{0.2}-\mathrm{PHI} / \mathrm{PHI}$ catalysts. The molecular structure of (b) PCN, (d) PHI and (f) $\mathrm{Zn}_{\mathrm{x}}-\mathrm{PHI}$. The gray, blue, white, and brown spheres denote $\mathrm{C}, \mathrm{N}, \mathrm{H}$, and $\mathrm{Zn}$ atoms, respectively. 


\section{Physicochemical property of $\mathrm{WO}_{3}$ and $\mathrm{BiVO}_{4}$}

(a)
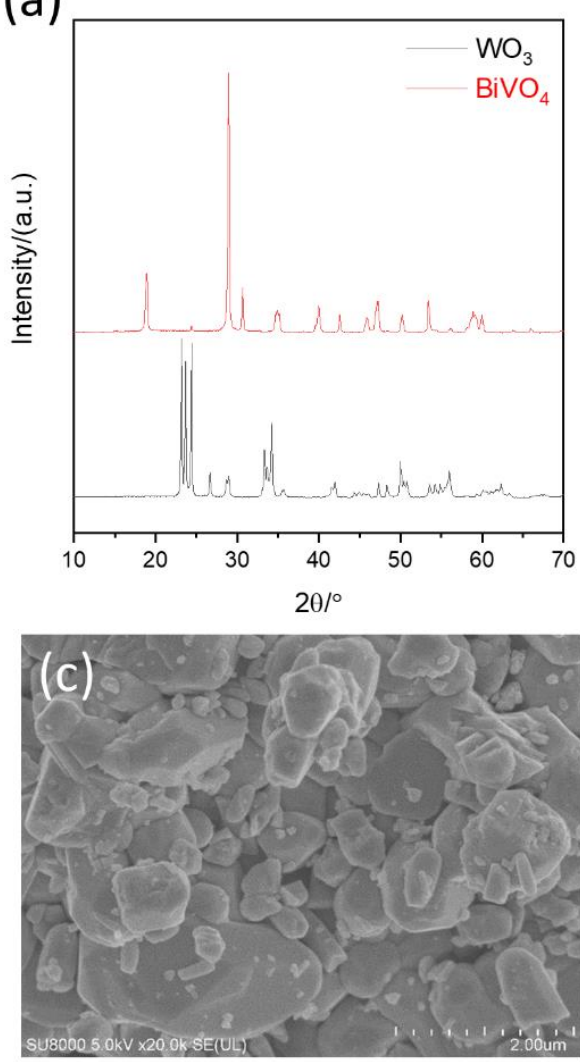

(b)
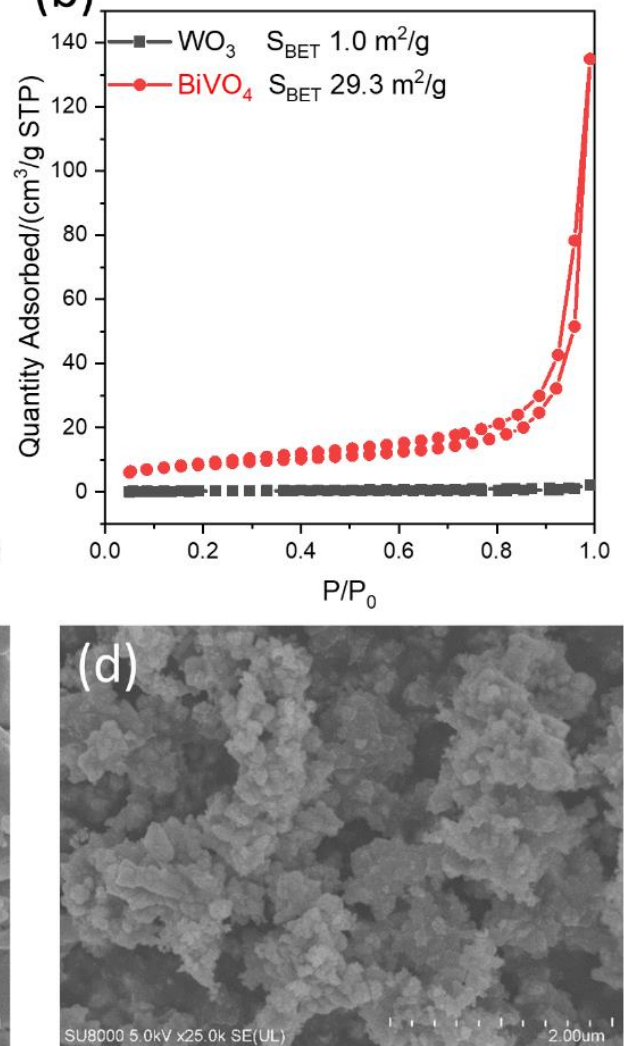

Figure S8. (a) XRD patterns and (b) Nitrogen adsorption-desorption isotherms of commercial $\mathrm{WO}_{3}$ and commercial $\mathrm{BiVO}_{4}$. The SEM images of (c) commercial $\mathrm{WO}_{3}$ and (d) commercial $\mathrm{BiVO}_{4}$. 


\section{Photocatalytic water oxidation experiment}
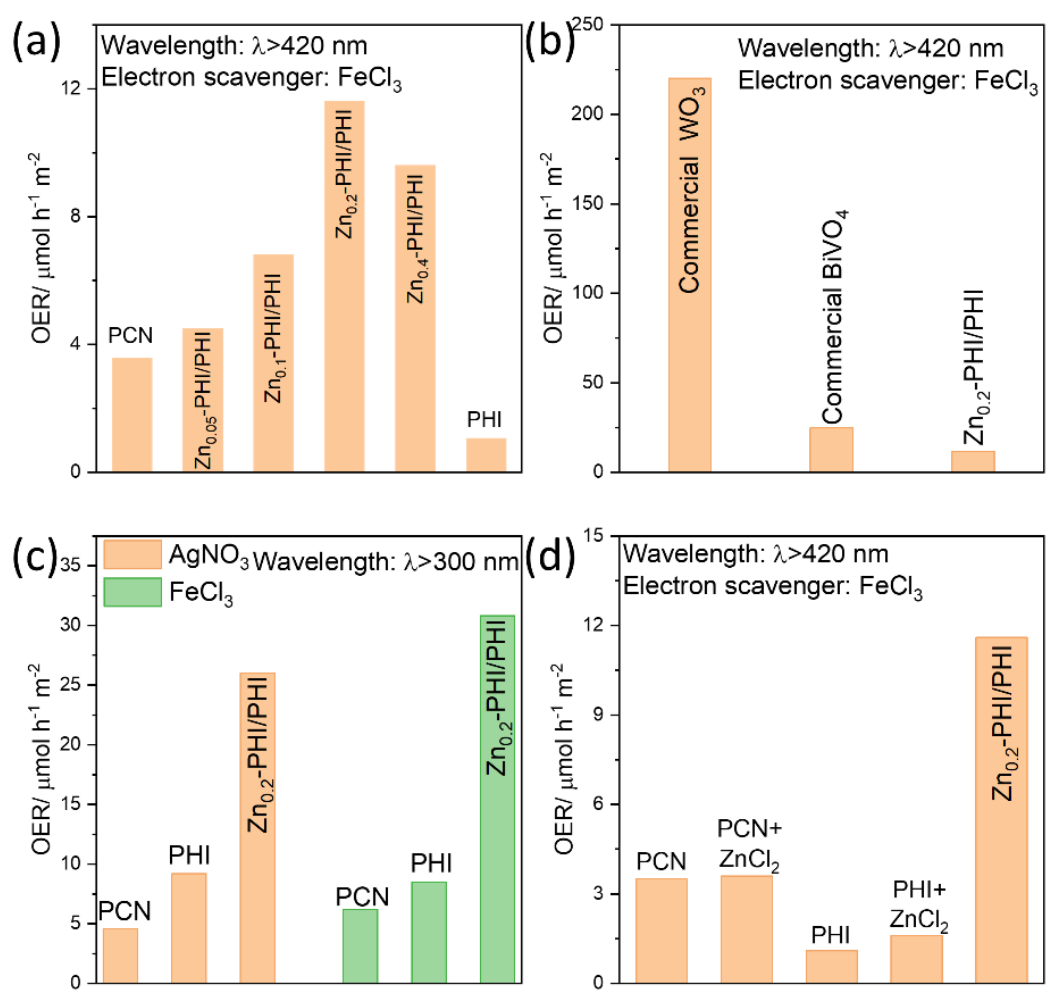

Figure S9. ( $\mathrm{a}$ and b) $\mathrm{O}_{2}$ evolution rates normalized with materials surface area of PCN, PHI, $\mathrm{WO}_{3}, \mathrm{BiVO}_{4}$ and $\mathrm{Zn}_{\mathrm{x}}-\mathrm{PHI} / \mathrm{PHI}$ samples under visible light $(\lambda>420 \mathrm{~nm})$ irradiation in the presence of $\mathrm{FeCl}_{3}(0.05 \mathrm{M})$ as an electron scavenger. (c) $\mathrm{O}_{2}$ evolution rates normalized with materials surface area of PCN, PHI, and $\mathrm{Zn}_{0.2}-\mathrm{PHI} / \mathrm{PHI}$ samples under visible light $(\lambda>420 \mathrm{~nm})$ irradiation in the presence of $\mathrm{FeCl}_{3}(0.05 \mathrm{M})$ or $\mathrm{AgNO}_{3}$ $(0.1 \mathrm{M})$ as electron scavenger; (d) $\mathrm{O}_{2}$ evolution rates normalized with materials surface area of different catalysts under visible light $(\lambda>420 \mathrm{~nm})$ irradiation in the presence of $\mathrm{FeCl}_{3}(0.05 \mathrm{M})$ as electron scavenger. 


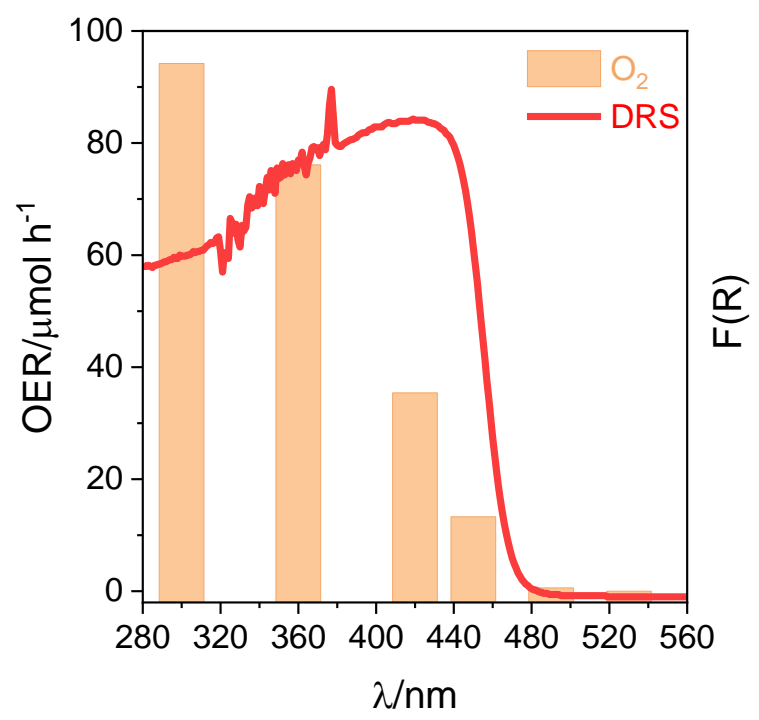

Figure S10. Wavelength dependence oxygen evolution rates of $\mathrm{Zn}_{0.2}-\mathrm{PHI} / \mathrm{PHI}$ catalyst for photocatalytic water oxidation in the presence of $\mathrm{Fe}^{3+}(0.05 \mathrm{M})$ as electron donor.

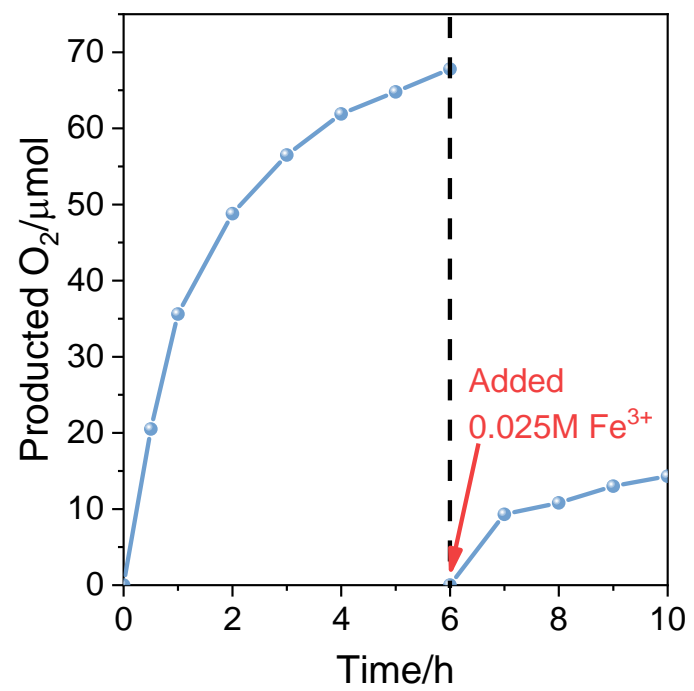

Figure S11. Time course $\mathrm{O}_{2}$ evolution of $\mathrm{Zn}_{0.2}-\mathrm{PHI} / \mathrm{PHI}$ catalyst for photocatalytic water oxidation in the presence of $\mathrm{Fe}^{3+}(0.05 \mathrm{M})$ as electron donor, $\lambda>420 \mathrm{~nm}$. 


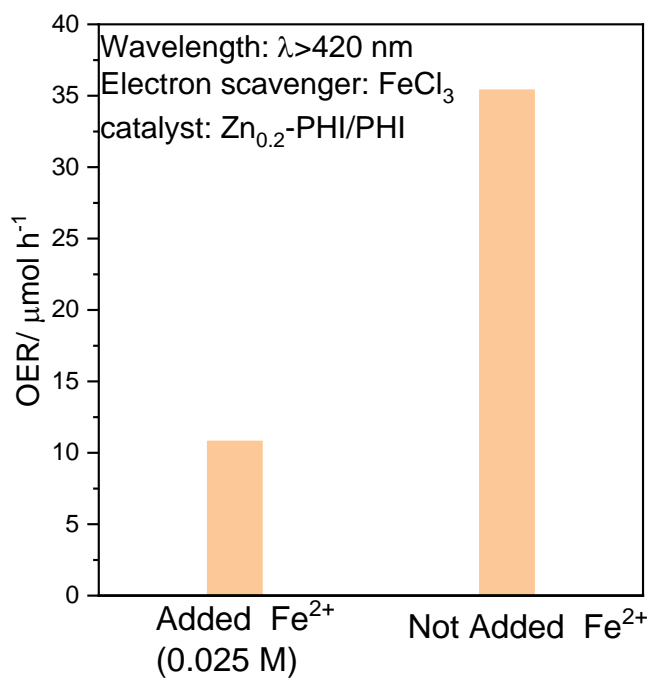

Figure S12. Photocatalytic water oxidation rates over $\mathrm{Zn}_{0.2}$-PHI/PHI under visible light $(\lambda>420 \mathrm{~nm})$ irradiation in the $0.05 \mathrm{M} \mathrm{Fe}^{3+}$ solution (or the $0.05 \mathrm{M} \mathrm{Fe}^{3+}$ and $0.025 \mathrm{M}$ $\mathrm{Fe}^{2+}$ mixed solution);

Physicochemical characterizations of after reaction of $\mathrm{Zn} 0.2-\mathrm{PHI} / \mathrm{PHI}$

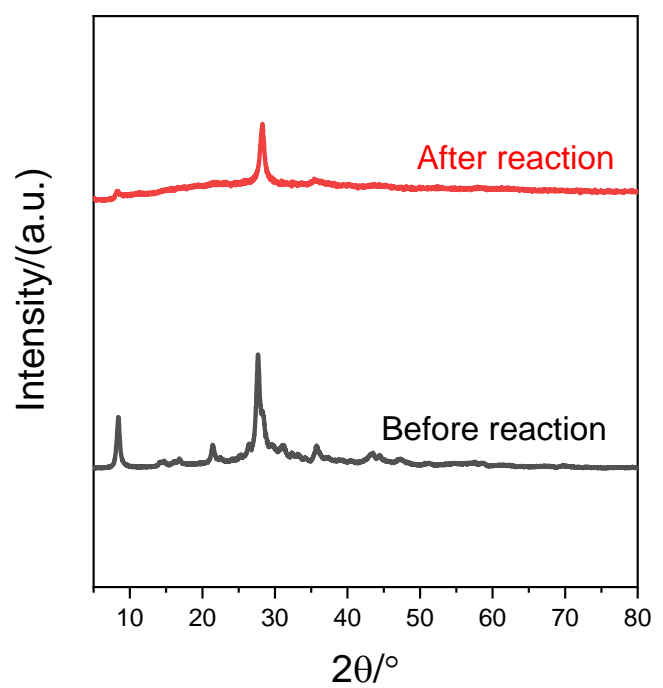

Figure S13. XRD of after reaction sample of $\mathrm{Zn}_{0.2}-\mathrm{PHI} / \mathrm{PHI}$ : fresh sample and used sample after the photocatalytic water oxidation reaction. 

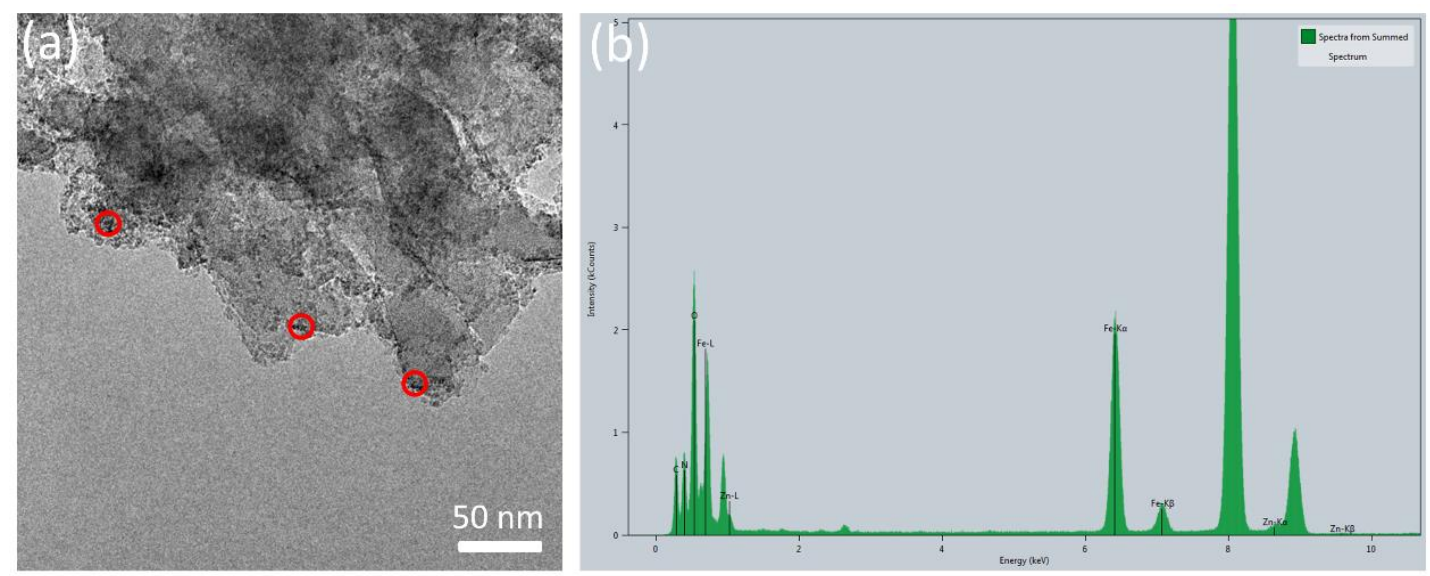

Figure S14. TEM images (a) and EDX spectrum (b) of after reaction sample of $\mathrm{Zn}_{0.2^{-}}$ PHI/PHI: used sample after the photocatalytic water oxidation reaction.

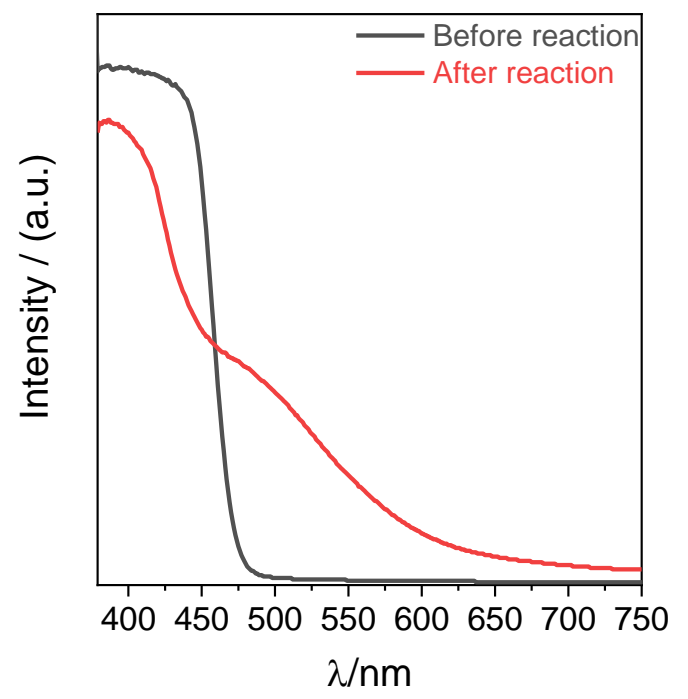

Figure S15. DRS of after reaction sample of $\mathrm{Zn}_{0.2}-\mathrm{PHI} / \mathrm{PHI}$ : fresh sample and used sample after the photocatalytic water oxidation reaction. 

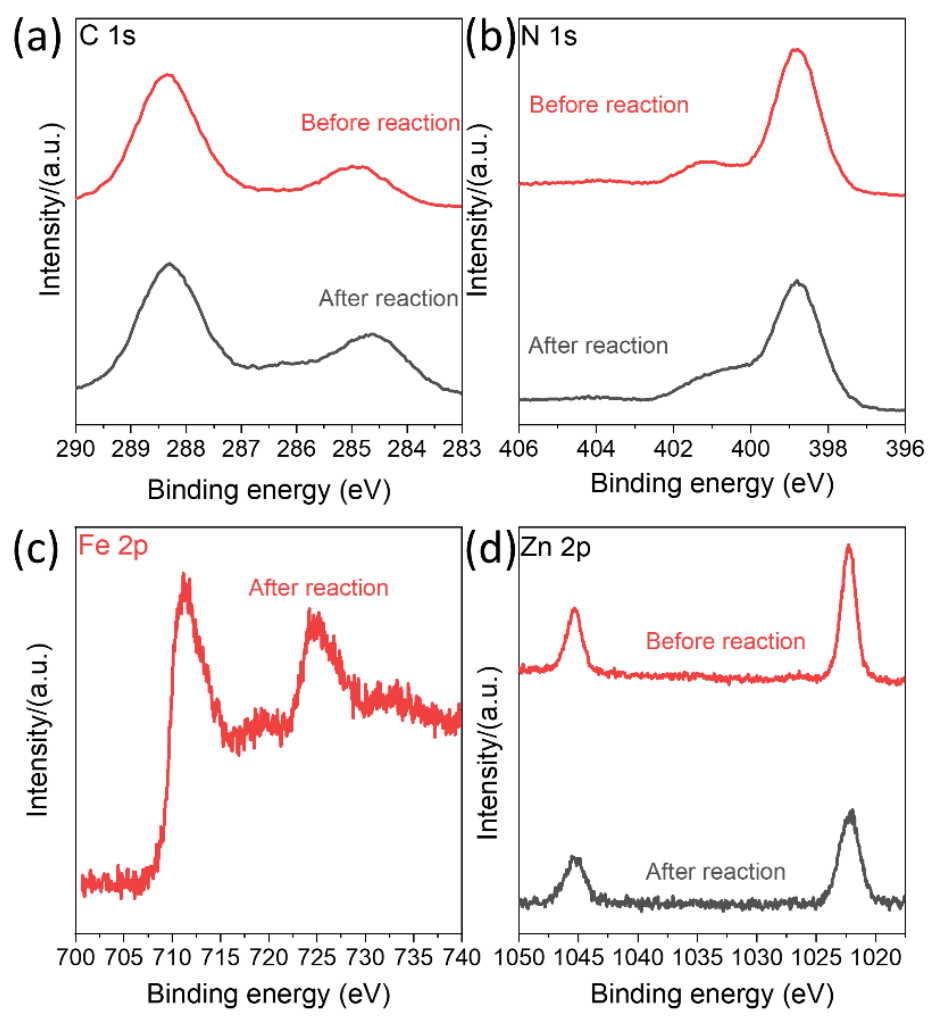

Figure S16. XPS of after reaction sample of $\mathrm{Zn}_{0.2}-\mathrm{PHI} / \mathrm{PHI}$ : fresh sample and used sample after the photocatalytic water oxidation reaction. 
Table S2. Comparison of PCN-base materials for photocatalytic OER.

\begin{tabular}{|c|c|c|c|c|}
\hline Materials & Reaction conditions & $\begin{array}{l}\mathrm{O}_{2} \text { evolution rate } \\
\left(\mu \mathrm{mol} \cdot \mathrm{h}^{-1}\right)\end{array}$ & $\operatorname{AQY}(\%)$ & Ref \\
\hline $3 \% \mathrm{Co}(\mathrm{OH})_{2} / \mathrm{BH} 400$ & $\begin{array}{l}300 \mathrm{~W} \text { Xe lamp, } 50 \mathrm{mg} \\
\text { catalyst, Aqueous } \\
\begin{array}{ll}\mathrm{AgNO}_{3} \\
\text { solution }(0.01 \mathrm{M})\end{array}\end{array}$ & $\begin{array}{l}28.1 \quad(\lambda>300 \mathrm{~nm}) \\
8.0 \quad(\lambda>420 \mathrm{~nm})\end{array}$ & $380 \mathrm{~nm}: 3.7$ & 2 \\
\hline $3 \% \mathrm{CoP} / \mathrm{PCN}$ & $\begin{array}{l}300 \mathrm{~W} \text { Xe lamp, } 50 \mathrm{mg} \text { catalyst, } \\
\text { Aqueous } \mathrm{AgNO}_{3} \text { solution } \\
(0.01 \mathrm{M})\end{array}$ & $42 \quad(\lambda>300 \mathrm{~nm})$ & N/A & 3 \\
\hline $10 \% \mathrm{CoMn}_{2} \mathrm{O}_{4} / \mathrm{PCN}$ & $\begin{array}{l}300 \mathrm{~W} \text { Xe lamp, } 50 \mathrm{mg} \text { catalyst, } \\
\text { Aqueous } \mathrm{AgNO}_{3} \text { solution } \\
(0.01 \mathrm{M})\end{array}$ & $18.3 \quad(\lambda>300 \mathrm{~nm})$ & $380 \mathrm{~nm}: 1.0$ & 4 \\
\hline $\begin{array}{l}1 \% \mathrm{NiCoP} / \mathrm{NiCo}- \\
\mathrm{Pi} / \mathrm{PCN}\end{array}$ & $\begin{array}{l}300 \mathrm{~W} \text { Xe lamp, } 50 \mathrm{mg} \text { catalyst, } \\
\text { Aqueous } \mathrm{AgNO}_{3} \text { solution } \\
(0.02 \mathrm{M})\end{array}$ & $15.6(\lambda>300 \mathrm{~nm})$ & $420 \mathrm{~nm}: 0.6$ & 5 \\
\hline $3 \% \mathrm{Co}(\mathrm{OH})_{2} / \mathrm{PCN}$ & $\begin{array}{l}300 \mathrm{~W} \text { Xe lamp, } 50 \mathrm{mg} \text { catalyst, } \\
\text { Aqueous } \mathrm{AgNO}_{3} \text { solution } \\
(0.01 \mathrm{M})\end{array}$ & $\begin{array}{ll}27.4 & (\lambda>300 \mathrm{~nm}) \\
7.1 & (\lambda>420 \mathrm{~nm})\end{array}$ & N/A & 6 \\
\hline $1 \%$ Co doped PCN & $\begin{array}{l}300 \mathrm{~W} \text { Xe lamp, } 50 \mathrm{mg} \text { catalyst, } \\
\text { Aqueous } \mathrm{AgNO}_{3} \text { solution } \\
(0.01 \mathrm{M})\end{array}$ & $13.0 \quad(\lambda>300 \mathrm{~nm})$ & N/A & 7 \\
\hline $3 \% \mathrm{CoSe} / \mathrm{PCN}$ & $\begin{array}{l}300 \mathrm{~W} \text { Xe lamp, } 50 \mathrm{mg} \text { catalyst, } \\
\text { Aqueous } \mathrm{AgNO}_{3} \text { solution } \\
(0.01 \mathrm{M})\end{array}$ & $\begin{array}{ll}34 & (\lambda>300 \mathrm{~nm}) \\
9 & (\lambda>420 \mathrm{~nm})\end{array}$ & N/A & 8 \\
\hline $3 \% \mathrm{Co}_{3} \mathrm{O}_{4} / \mathrm{HR}-\mathrm{PCN}$ & $\begin{array}{l}300 \mathrm{~W} \text { Xe lamp, } 50 \mathrm{mg} \text { catalyst, } \\
\text { Aqueous } \mathrm{AgNO}_{3} \text { solution } \\
(0.01 \mathrm{M})\end{array}$ & $(\lambda>300 \mathrm{~nm})$ & N/A & 9 \\
\hline $\mathrm{CNS}_{650}$ & $\begin{array}{l}300 \mathrm{~W} \text { Xe lamp, } 50 \mathrm{mg} \text { catalyst, } \\
\text { Aqueous } \mathrm{AgNO}_{3} \text { solution } \\
(0.01 \mathrm{M})\end{array}$ & $20.1 \quad(\lambda>300 \mathrm{~nm})$ & N/A & 10 \\
\hline $0.3 \% \mathrm{CoO}_{\mathrm{x}}-\mathrm{PCN}-\mathrm{Br}_{0.1}$ & $\begin{array}{l}300 \mathrm{~W} \text { Xe lamp, } 50 \mathrm{mg} \text { catalyst, } \\
\text { Aqueous } \mathrm{AgNO}_{3} \text { solution } \\
(0.01 \mathrm{M})\end{array}$ & $\begin{array}{ll}23 & (\lambda>300 \mathrm{~nm}) \\
4 & (\lambda>420 \mathrm{~nm})\end{array}$ & N/A & 11 \\
\hline $\mathrm{Zn}_{0.2}-\mathrm{PHI} / \mathrm{PHI}$ & $\begin{array}{l}300 \mathrm{~W} \text { Xe lamp, } 50 \mathrm{mg} \text { catalyst, } \\
\text { Aqueous } \mathrm{AgNO}_{3} \text { solution } \\
(0.01 \mathrm{M})\end{array}$ & $79.3 \quad(\lambda>300 \mathrm{~nm})$ & N/A & $\begin{array}{l}\text { This } \\
\text { work }\end{array}$ \\
\hline $\mathrm{Zn}_{0.2}-\mathrm{PHI} / \mathrm{PHI}$ & $\begin{array}{l}300 \mathrm{~W} \text { Xe lamp, } 50 \mathrm{mg} \text { catalyst, } \\
\text { Aqueous } \quad \mathrm{FeCl}_{3} \text { solution } \\
(0.005 \mathrm{M})\end{array}$ & $\begin{array}{ll}94.2 & (\lambda>300 \mathrm{~nm}) \\
35.4 & (\lambda>420 \mathrm{~nm})\end{array}$ & $420 \mathrm{~nm}: 3.6$ & $\begin{array}{l}\text { This } \\
\text { work }\end{array}$ \\
\hline
\end{tabular}




\section{Optical characterization}

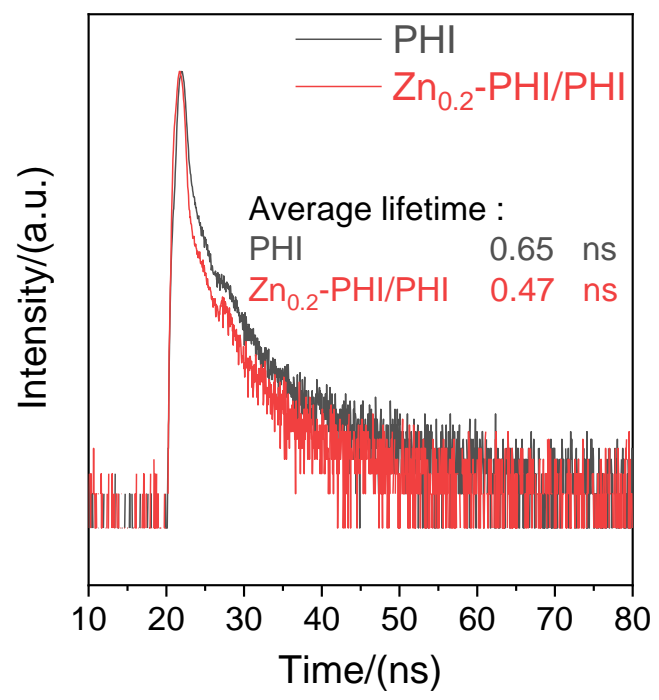

Figure S17. Time-resolved PL of PHI and $\mathrm{Zn}_{0.2}-\mathrm{PHI} / \mathrm{PHI}$ catalysts. 

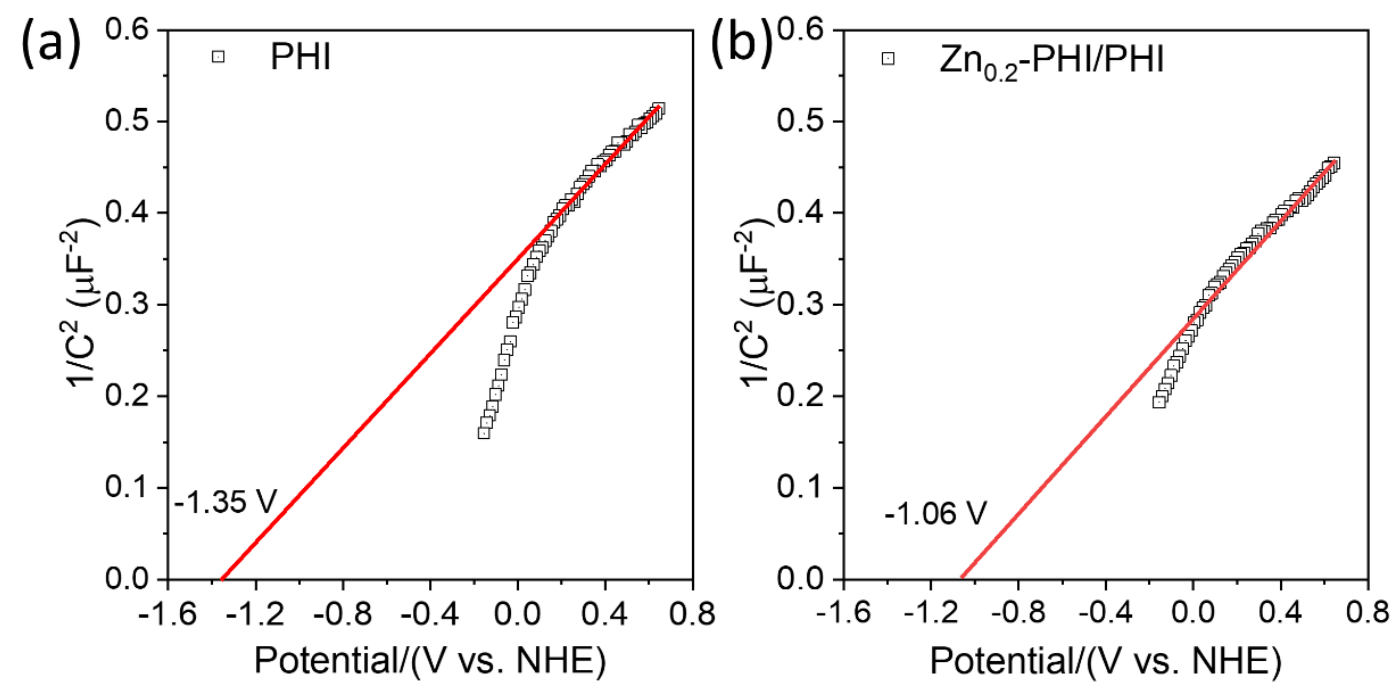

Figure S18. Mott-Schottky plots of (a) PHI and (b) $\mathrm{Zn}_{0.2}-\mathrm{PHI} / \mathrm{PHI}$ catalysts.

Table S3. EIS fitted parameters of PHI and $\mathrm{Zn}_{0.2}-\mathrm{PHI} / \mathrm{PHI}$ catalyst shown in Figure 7a.

The equivalent circuit parameters include: the equivalent circuits consist of series resistance $\left(R_{1}\right)$; the charge tranfer resistance $\left(R_{2}\right)$; the constant phase element $\left(C_{2}\right)$.

\begin{tabular}{l|l|l|l}
\hline Catalysts & $\mathrm{R}_{1}$ & $\mathrm{R}_{2}$ & $\mathrm{C}_{2}$ \\
\hline $\mathrm{PHI}$ & $47.7 \Omega$ & $33211 \Omega$ & $2.63 * \mathrm{e}^{-6} \mathrm{~F}$ \\
\hline $\mathrm{Zn}$ 0.2-PHI/PHI & $43.2 \Omega$ & $20279 \Omega$ & $3.51 * \mathrm{e}^{-6} \mathrm{~F}$ \\
\hline
\end{tabular}




\section{REFERENCES}

1. Li, X.; Wu, Y.; Zhang, S.; Cai, B.; Gu, Y.; Song, J.; Zeng, H., CsPbX 3 Quantum Dots for Lighting and Displays: Room-Temperature Synthesis, Photoluminescence Superiorities, Underlying Origins and White Light-Emitting Diodes. Adv. Funct. Mater. 2016, 26, 2435-2445.

2. Zhao, D.; Dong, C.-L.; Wang, B.; Chen, C.; Huang, Y.-C.; Diao, Z.; Li, S.; Guo, L.; Shen, S., Synergy of Dopants and Defects in Graphitic Carbon Nitride with Exceptionally Modulated Band Structures for Efficient Photocatalytic Oxygen Evolution. Adv. Mater. 2019, 31, 1903545.

3. Pan, Z.; Zheng, Y.; Guo, F.; Niu, P.; Wang, X., Decorating CoP and Pt Nanoparticles on Graphitic Carbon Nitride Nanosheets to Promote Overall Water Splitting by Conjugated Polymers. ChemSusChem 2017, 10, 87-90.

4. Zhang, L.; Yang, C.; Xie, Z.; Wang, X., Cobalt Manganese Spinel as an Effective Cocatalyst for Photocatalytic Water Oxidation. Appl. Catal., B 2018, 224, 886-894.

5. Qin, Z.; Chen, Y.; Huang, Z.; Su, J.; Guo, L., A Bifunctional NiCoP-Based Core/Shell Cocatalyst to Promote Separate Photocatalytic Hydrogen and Oxygen Generation over Graphitic Carbon Nitride. J. Mater. Chem. A 2017, 5, 19025-19035.

6. Zhang, G.; Zang, S.; Wang, X., Layered $\mathrm{Co}(\mathrm{OH})_{2}$ Deposited Polymeric Carbon Nitrides for Photocatalytic Water Oxidation. ACS Catal. 2015, 5, 941-947.

7. Zhang, G.; Huang, C.; Wang, X., Dispersing Molecular Cobalt in Graphitic Carbon Nitride Frameworks for Photocatalytic Water Oxidation. small 2015, 11, 1215-1221.

8. Zhang, G.; Zang, S.; Lan, Z.-A.; Huang, C.; Li, G.; Wang, X., Cobalt Selenide: A Versatile Cocatalyst for Photocatalytic Water Oxidation with Visible Light. J. Mater. Chem. A 2015, 3, 17946-17950.

9. Zheng, Y.; Lin, L.; Ye, X.; Guo, F.; Wang, X., Helical Graphitic Carbon Nitrides with Photocatalytic and Optical Activities. Angew. Chem., Int. Ed. 2014, 53, 1192611930.

10. Zhang, J.; Sun, J.; Maeda, K.; Domen, K.; Liu, P.; Antonietti, M.; Fu, X.; Wang, X., Sulfur-Mediated Synthesis of Carbon Nitride: Band-Gap Engineering and Improved Functions for Photocatalysis. Energy Environ. Sci. 2011, 4, 675-678.

11. Lan, Z.-A.; Zhang, G.; Wang, X., A Facile Synthesis of Br-Modified g- $\mathrm{C}_{3} \mathrm{~N}_{4}$ Semiconductors for Photoredox Water Splitting. Appl. Catal., B 2016, 192, 116-125. 